\title{
Design and Study of Logistics Management System Based on E-Commerce
}

\author{
Li Zhu \\ College of Mathematics and Computer Science \\ Jiangxi Science and Technology Normal University \\ Nanchang, China \\ E-mail: $131845722251 @ 163 . c o m$
}

\author{
Lan Bai \\ Computer and information branch \\ Jiangxi Modern Polytechnic College \\ Nanchang, China \\ E-mail: ilovexz0802@163.com
}

\begin{abstract}
This paper carries out analysis from the development situation of logistics management which highlights the process, technique of electronic commerce logistics, logistics management and techniques, logistics system transit lines. Besides, this paper specifically explains relevant software techniques which can help to realize logistics system development, including Struts structure and theory, three-layer B/S structure. At present, the logistics management develops quickly, and enterprise logistics has entered into a new era of e-commerce management. Adopting existing software development technology to research the enterprise logistics management software development will greatly accelerate the development of enterprise logistics.
\end{abstract}

Keywords-E-commerce; Logistics Management; $\quad B / S$ Structure; System Module; Struts Structure

\section{INTRODUCTION}

Nowadays, scientific information provides a new development opportunity for enterprises, and any enterprise is granted with the opportunity to contact customers throughout the world to establish business relation. The effective utilization of information has become an important means for enterprises to strengthen competence in new economic module and e-commerce has become a basic trading and communication means. By the way, the most significant effect which logistics industry has brought about is the change of e-commerce module and its development will re-define the logistics and shall ignite positive effects on all of the sectors as well as aspects of logistics. Enterprises of all fields are connected with each other via network. Moreover, enterprises in the same supply chain will become a concerted cooperation partner and employees are able to participate in the business process so as to provide better service for customers with totally new approaches.

\section{CURRENT ANALYSIS ON LOGISTICS MANAGEMENT}

Globally, transnational corporation such as IBM has already established business relations with famous logistics enterprises such as UPS, Fedex and Ryder; In China, IBM has various cooperation partners such as Ministry of Railways, Shanghai South Integrated Logistics Center etc. At present, the logistics industry in China has broader development space and huge market potential, which will become an important industry for the economic development in China. What's more, China has established a comprehensive transportation system integrating railway, highway, waterway, aviation and pipelines. However, it starts late in China with lagging concept as well as management system. Therefore, various sectors of the logistics process are separate and scattered, which has a gap comparing with integrated modern logistics industry.

How to change traditional logistics industry to modern logistics management module has become an urgent problem remaining to be solved. Therefore, only by adopting advanced information management means and integrating all sectors in the traditional logistics process, strengthening combination and management to create proper and transparent business process can researchers realize the development from traditional logistics to modern logistics.

\section{PROCESS AND TECHNOLOGY ELECTRONIC COMMERCE LOGISTICS}

\section{A. The logistics management of e-commerce}

The logistics management of e-commerce includes logistics system, logistics process and logistics technology which must realize the following targets: high-level enterprise management; high-quality personnel management; high-quality equipment deployment.

The logistics operation process of e-commerce is similar to common one because both of them aim to send the goods booked by customers to them and they have similar operation process, including package, goods transportation, storage, goods loading and unloading as well as logistics information management.

\section{B. The logistics technology of e-commerce}

E-commerce logistic technology aims to deliver and store the goods and materials and provide intangible service, including hardware technology and software technology:

Hardware technology includes:

(1) Basic infrastructure closely related to e-commerce logistics;

(2) Mechanical technology: Loading and unloading machines, sorting machinery, packaging machinery and transport machinery etc;

(3) Materials Technology:Packing material etc; 
(4) Logistics information technology and communication technology: it provides a series of computer controlling and network communication technology from original material to products;

Software technology includes:

(1) Planning technology: Carry out planning research and optimization as well as improvement on circulation form and hard technology.

(2) Transportation technology: The selection and utilization for transportation tools, loading and unloading approaches, inventory management, resource and labor service etc.

(3) Evaluation technology: Cost controlling and calculation as well as systemperformance evaluation.

\section{Logistics system transit line analysis}

Transit line analysis is to analyze the transport activities from the starting point to ending point which focuses on the balance. In order to maximize the transportation tools the transportation between the two points should be balanced or relatively balanced and the transit line should contain two or more points. Transit analysis is an important part of transportation logistics and there are four problems to be summed up: transportation problem, assignment problem, shortest route problem, travel route problem. In this paper, researchers briefly introduce the travel route problem in the following contents:

The transportation from one logistic point to another point (one sector in the logistics operation process) is an important element to construct the logistics cost. Supposing that the products are calculating based on pieces, and if the transportation tool is full with goods both in the delivery process or returning process, the

efficiency of logistics system shall be excellent. Under most conditions, such logistics transportation cannot be realized and there should be the condition that the freight is not full or is empty because of different transportation location or quantities. Therefore, researchers should optimize the shortest route. The following approaches can be adopted:

Firstly, from the orig inal point, the closest point can be regarded as the second point and so on until the ending point. However, this approach does not take the distance from the original point to the ending point which cannot properly handle with the situation of point of divergence (delivery from one point to several points) and assemblage point (delivery from several points to one point).

Secondly, from the original point, the closest point can be regarded as the second point and another closest point are the last but one point and so on to find the third point. Then, starting from the last but one point, researchers can take the closest point as the last but two points (the overlap place is not taken into consideration except for the last point). This approach can optimize the route yet is not the optimal solution.

\section{SYSTEMATIC B/S STRUCTURE}

The design of the software system structure is the key point in the course of developing the software. Different systems require different structural systems and the design of system largely depends on the selection of system structure. This system adopts B/S network structural system. The systematic B/S system structure shows in Fig. $1:$

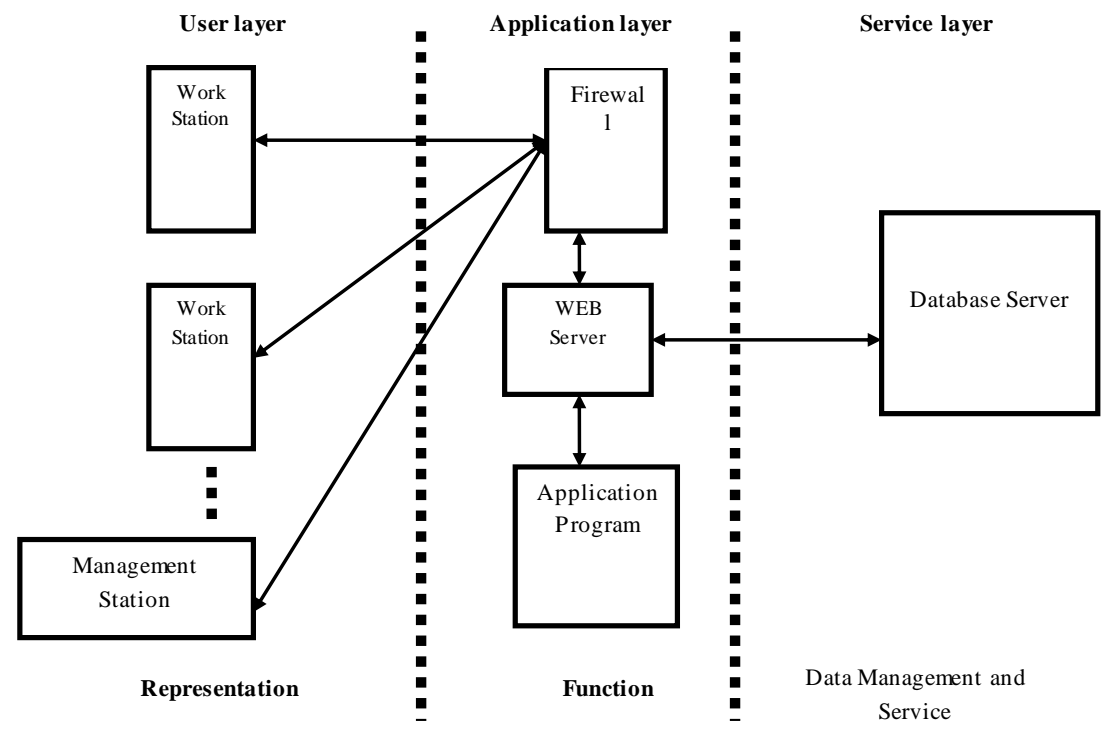

Figure 1. Systematic three layer B/S structure

$\mathrm{B} / \mathrm{S}$ structure divides three elements (data, function and behavior) to form front tier which is responsible for portable logical expression; the application layer allows customers to separate the design application so as to share and control the business logic; the back-end design separates the service layer and provides opportunity to deal with the data flow between client and database. B/S structure is a real three-layer structure, which focuses on viewing WEB database and HTTP is the transport protocol. In addition, the client side views the WEB server as well as the back-end database, which researchers call B/S (Browser/Server), and its merits show as follows:

Firstly, the network application system is crossplatform with good compatibility, which can protect the original software and hardware facilities. Therefore, the 
original network, operation system, database can easily adopt it to create better performance in the shortest time.

Secondly, researchers do not have to develop special client software or add any code on the client. Users can adopt the current browser with simple steps which is easy to learn and accept.

Thirdly, the technology is relatively mature with less investment. The system is easy to maintain with stable operation, which are safe and can be developed.

\section{V.DYNAMIC PROCESS ANALYSIS ON THE SYSTEM FUNCTION}

The logistics system is a huge system. Even though the logistics sector does not produce product value, it can determine the required technology indicators based on project needs:

Firstly, good interactivity: the work content is mainly about man-machine communication which requires good interactivity ability.

Secondly, better expansibility: the work content and form is changeable which requires better expansibility.

Thirdly, good maintainability: after the system is put into use, the administrator is responsible for the maintenance work and the administrator is regularly changing which asks good maintainability.
Fourthly, good cross-platform ability: users can adopt different operation systems. In order to be adapted to the potential changes, the system should possess good crossplatform ability.

Based on above four points, researchers adopt J2EE programming environment as well as the Struts structure while developing software module.

To well implement Struts structure project, the key is to possess a complete module and make it clear how many modules the systems possess and which kind of FormBean, JavaBean as well as results can realize the JSP. Meanwhile, researchers should also set strutsconfig. Xml document.

The basic components for J2EE to implement the project show as follows:

$\diamond \quad$ form stores ActionForm category

$\diamond \quad$ action stores Action

$\diamond \quad$ bean stores business logic category

$\diamond \quad$ data stores database table or data related to query results

$\diamond \quad$ util stores common tools

$\diamond \quad \mathrm{db}$ stores SQL as well as tools

Based on the login work flow, researchers can understand the basic human-machine interactive work flow contents and the system design module show in Fig. 2:

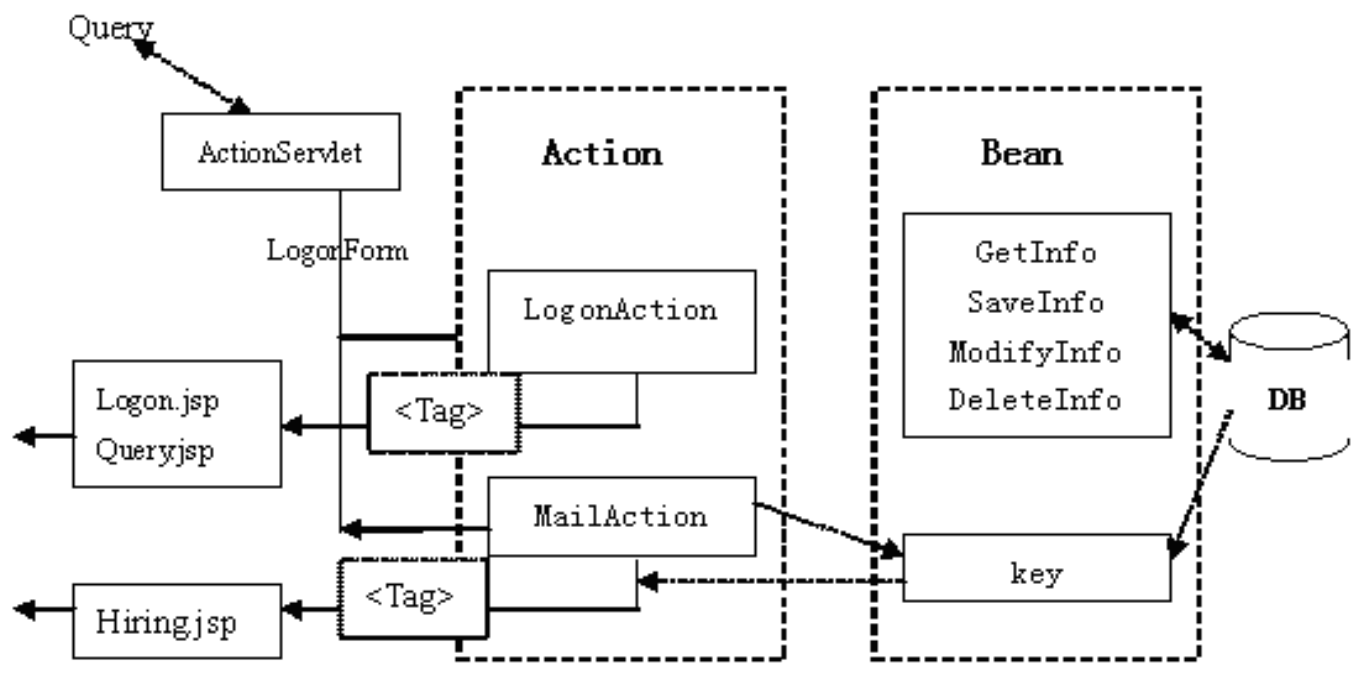

Figure 2. system design module

ActionServlet accepts all requirements of HTTP and then based on the contents of profiles it determines to reflect the Action objects. There are two Action objects in this systemwhich is LogoAction and MailAction.

LogoAction firstly verify whether users have entered into the system or not and if they have not entered in, it will turn back to the login page (Logon.jsp). After the verification, if users have selected the rent software, it turns to Query.jsp. If users select some software or modules the MailAction shall send passwords to them once they submit the information. Once accepting the pass word, they can login.

If users successfully login, it can read the rent software information and put it into FormBean based on the ActionForward in struts-config. Xml. Then, this system can demonstrate the Bean data by calling the JSP page. If it suggests to store the information, this system will call SaveInfo to store the data from the FormBean and if it suggests to modify the information, it call Modifyinfo to modify the information and then store it; if it suggests to delete the information, this system call DeleteInfo to delete the data kept in the FormBean.

Based on such system, the user interface and data processing have been completely separated. What's more, with the custom tags in the JSP page, there is no Java script code. As a result, the design of Web and the writing of the back program have a clear boundary, which is easy to develop and maintain. 


\section{SYSTEM MODULE DIVISION}

Business management system can be generally divided into the following subsystems: procurement systems, delivery systems, marketing systems, contract management, integrated WEB management system.

The database is divided into space database, database platform and the GIS platform, among which when researchers acquire a certain property from the space database for example, the branch name, suppliers name, warehouse name, and administrative divisions name researchers can easily obtain its property data. Besides, any GIS platform has its database engine which can view its established spatial database. Sales system is located on the branch end, so this paper designs and develops the purchasing system, delivery system, and contract management system, trying to solve practical problems with its theories.

The application system of three layer B/S structure has lower requirements on the client, which will not bring along with troubles for the software upgrading which can guarantee the coordination and unification of JSP. As a result, the system structure is clear and can be easily maintained which is able to properly separate the front and back so as to guarantee the system security.

\section{CONCLUSION}

Modern logistics is a complex system-project, which is closely related with various industries. Along with the further development of e-commerce logistics, its management shall develop from single type to multiple types. The logistics management system is a product of the information age, and with the development of information technology, logistics software will become popular. Besides, the logistics management will be further refined and shall be widely applied.

\section{REFERENCES}

[1] An Shumin etc. Network equipment and management [M].Beijing : Beijing hope electronic press, $2013: 25-38$

[2] Wang Qun etc. Electronic logistics management for E-commerce Business [M].Beijing: Science Press, 2002.

[3] Xia Yang. Web application based on Struts system structure[J]. Computer and Modernization

[4] Wu Qing, Liu Jia. Customer relationship management[M]. Shanghai: Fudan University Press.

[5] Wang Donggang. Software test and JuniT practice [M]. Posts \& Telecom Press, 2010: 32 - 40

[6] Mao Guojun etc. Principle and Algorithm of Data Mining [M]. Beijing: T singhua University Press

[7] Gao Minfang. Analysis on the problem and countermeasure of ecommerce logistics delivery in China [J]. China Business \& Trade, 2011(33)

[8] Liu Zhaohui. Study on e-commerce logistics system[J]. Value Engineering. 2011(13)

[9] Wang Xiaoling, Zhang Baoming. Elect ronic purchasing advantages and strategic selection [J].Logistics Management. 2011(04)

[10] Liu Yuantao, Wang Junmei. Lean Logistics-Effective means to decrease storage [J]. Logistics \& Material Handling. 2010(10)

[11] Tan Zheng, Cao Yuhui. M-Commerce[M]. Beijing: Tsinghua University Press, 2012.113-121.

[12] Lv Tingjie. M-Commerce [M]. Beijing: Publishing House of Electronics Industry, 2011.313-316 